\title{
Distribuição e diversidade de espécies do gênero Biomphalaria em microrregiões localizadas no Médio Paranapanema, São Paulo, SP, Brasil
}

\author{
Roseli Tuan ${ }^{1,2}$ \\ ${ }^{1}$ Laboratório de Bioquímica e Biologia Molecular, \\ Superintendência de Controle de Endemias - SUCEN, \\ Secretaria da Saúde do Estado de São Paulo, \\ Rua Paula Souza, 166, Luz, CEP 01027-000, São Paulo, SP, Brasil \\ ${ }^{2}$ Autor para correspondência: RoseliTuan,e-mail: roselituan@yahoo.com.br
}

TUAN, R. 2009. Diversity and distribution of the Biomphalaria species in the middle reaches of the Paranapanema River, São Paulo, SP, Brazil. Biota Neotrop. 9(1): http://www.biotaneotropica.org.br/v9n1/en/ abstract?short-communication+bn03009012009.

\begin{abstract}
The predominant landscape in the studied region, composed by the Paranapanema and Pardo rivers, has been significantly affected by human interference in order to create extensive artificial irrigation ditches for agriculture. These environments are subject to drastic variations in water supply whereby draughts are created what bears a drastic populational fluctuation. The composition of schistosomiasis-associated planorbid fauna in Ourinhos and Ipauçu herein presented corroborate previous malacological surveys, which incriminates Biomphalaria. glabrata (Say, 1818) as the predominant species mainly at the Ourinhos micro-regions. At this site the snail distribution might be linked to the composition of deposited sediments in the hydrographic basin. Data from Ipauçu, a collection site that is only $30 \mathrm{~km}$ from Ourinhos, indicates absence of B. glabrata, and predominance of B. tenagophila (Orbigny, 1835). Despite closeness, the micro-regions show important differences in values of diversity index, indicating a heterogeneous distribution of Biomphalaria species.
\end{abstract}

Keywords: Biomphalaria, diversity, distribution, schistosomiasis.

TUAN, R. 2009. Diversidade e distribuição de espécies do gênero Biomphalaria em microrregiões localizadas no Médio Paranapanema, São Paulo, SP, Brasil. Biota Neotrop. 9(1): http://www.biotaneotropica.org.br/v9n1/ pt/abstract?short-communication+bn03009012009.

Resumo: Foram estudadas a diversidade e a abundância de espécies do gênero Biomphalaria em córregos próximos aos Rios Paranapanema e Pardo (São Paulo, SP, Brasil), em locais antigamente associados à transmissão do Schistosoma mansoni, sujeitos ainda a drásticas variações na disponibilidade de água. Os dados confirmam a predominância de Biomphalaria glabrata (Say, 1818) em córregos do município de Ourinhos, localizados nas margens do Rio Pardo e do Rio Paranapanema. Em Ipauçu, distante $30 \mathrm{~km}$ de Ourinhos, a predominância de Biomphalaria tenagophila (Orbigny, 1835) é acompanhada da ausência de B. glabrata. Foram estimados os índices de Diversidade e Dominância de Simpson, que evidenciam uma distribuição variada, provavelmente associada com o substrato aquático onde vivem os caramujos.

Palavras-chave: Biomphalaria, diversidade, distribuição, esquistossomose. 


\section{Introdução}

No Estado de São Paulo são encontradas dez das quatorze espécies de Biomphalaria descritas na região Neotropical (Estrada et al. 2006), incluindo as três espécies hospedeiras intermediárias do Schistosoma mansoni: Biomphalaria glabrata (Say 1818), Biomphalaria tenagophila (Orbigny 1835) e Biomphalaria straminea (Dunker 1848). Os caramujos habitam áreas naturais ou corpos d'água artificiais, freqüentemente efêmeros, sujeitos a secas periódicas. Via de regra, ciclos de cheia-seca são perturbações em ecossistemas com influência na variação da diversidade de espécies (Buckling et al. 2000). Os caramujos do gênero Biomphalaria apresentam duas características biológicas fundamentais para a preservação e a expansão das espécies e populações, em ambientes sujeitos as perturbações ambientais: 1) são hermafroditas simultâneos e se reproduzem tanto por fecundação cruzada, quanto por autofecundação (Paraense 1955); e 2) em situações de seca, dessecam, mantendo-se vivos, recolhidos à concha, em estágio fisiológico vegetativo, preservando-se até a próxima estação úmida. (Tuan \& Simões 1989). Apesar de o parasitismo afetar drasticamente os caramujos, a abundância, a distribuição e a diversidade das espécies podem ser mais fortemente alteradas pelo modo de reprodução e dessecação, do que por infestação por parasitas (Gerard et al. 2007).

O objetivo deste estudo é complementar os dados existentes de distribuição de espécies de Biomphalaria em antigos focos de esquistossomose, localizados na porção média do Rio Paranapanema, em ambientes caracterizados por drásticas alterações locais provocadas pelo homem. Neste caso, a fragmentação dos ecossistemas de água doce, manejados para atender as necessidades humanas, pode estar diretamente relacionada com a distribuição de espécies de Biomphalaria. Na primeira fase do estudo descrevemos a significativa diversidade genética, especialmente de Biomphalaria tenagophila, em criadouros adjacentes, localizados entre os Rios Paranapanema e Pardo, antigos focos de esquistossomose (Tuan \& Santos 2007). Complementa tal estudo a analise de aspectos quantitativos da diversidade de Biomphalaria, para inferir sobre as variáveis que condicionam a permanência e a estabilidade de populações de caramujos em coleções d'água sujeitas a perturbações hidrológicas.

\section{Material e Métodos}

As espécies de Biomphalaria foram coletadas em córregos que abastecem de água extensas hortas no município de Ourinhos $\left(22,98^{\circ} \mathrm{W}\right.$ e $\left.49,87^{\circ} \mathrm{S}\right)$, localizados entre o Rio Pardo e Paranapanema, e em valas no município de Ipauçu $\left(23,05^{\circ} \mathrm{W}\right.$ e $\left.49,62^{\circ} \mathrm{S}\right)$, localizado nas margens do Rio Paranapanema e distante $30 \mathrm{~km}$ de Ourinhos (Figura 1).

Foram realizadas quatro coletas consecutivas de caramujos na extensão total das localidades citadas, entre julho de 2003 e novembro de 2004. Para a coleta de caramujos foram utilizadas conchas de captura padronizadas para uso no Programa de Controle da Esquistossomose (Ministério da Saúde 2007).Para a pesquisa de cercárias, os caramujos de cada ponto de coleta foram reunidos, em grupos de até 10 indivíduos, em frascos de vidro transparente, com água filtrada, e expostos à luz de lâmpadas incandescentes (de $60 \mathrm{~W}$ ), durante no mínimo 4 horas. A visualização das cercárias, quando eliminadas na água, é feita com o auxílio do microscópio estereoscópico (Ruiz, 1952). Uma vez que o resultado da pesquisa de cercarias na água resultou negativo para todos os grupos de caramujos, nenhum caramujo foi examinado através de esmagamento de suas conchas.

A identificação específica de Biomphalaria fez-se através da morfologia de órgãos internos, especialmente aquelas do aparelho reprodutor feminino e masculino (Deslandes 1959, Paraense
1975, 1981). Foram estimados os seguintes parâmetros para uma descrição da fauna de caramujos do gênero Biomphalaria:

Frequência, pi $=n_{x} / \mathrm{N}$, onde $n i$ é o número de indivíduos de uma espécie x e $N$ o número total de exemplares coletados;

Índices de Dominância (Ds) e Diversidade de Simpson (Simpson 1949), 1s, relacionados com a probabilidade de dois indivíduos coletados ao acaso na mesma localidade pertencerem a mesma espécie; Índice Hill modificado (E), que estima a equitabilidade, ou seja, a distribuição dos indivíduos entre as espécies coletadas numa localidade. O índice Hill modificado tem como parâmetros os índices de Simpson e Shanon (Shanon 1948).

Os índices foram calculados através das rotinas implementadas no programa DivEs (Lizaro Soft, Rodrigues 2005).

\section{Resultados e Discussão}

A composição da fauna planorbídica nas valas e córregos da região de Ourinhos é alvo de constante monitoramento, desde o ano de 1952, período em que a esquistossomose representava um problema de saúde pública para a população local (Rey 1952). Desde então, sabia-se que a espécie predominante, $B$. glabrata, era altamente suscetível ao S. mansoni (Magalhães \& Dias 1973). Estudos posteriores mostravam que a predominância de $B$. glabrata implicava na baixa frequiência de $B$. tenagophila, supostamente por exclusão competitiva (Kawazoe et al. 1980). Em 1981, Paraense descreve a B. occidentalis, esclarecendo definitivamente as diferenças específicas entre a espécie e a B. tenagophila. Refeito o quadro de composição de planorbídeos na região de Ourinhos, deu-se como certa a presença de $B$. glabrata, B. tenagophila e B. occidentalis (Piza et al 1972, Vaz et al. 1985, Teles \& Vaz, 1987, Teles 2005).

Os resultados deste estudo corroboram estes dados e confirmam a predominância de B. glabrata na macro região que compreende os municípios de Ourinhos e Ipauçu (Figura 2).

Contudo, mesmo em áreas adjacentes, observamos que a distribuição de espécies de Biomphalaria é heterogênea (Figura 3). Em Ourinhos, a maior riqueza de espécies está no córrego Christone, onde B. glabrata e B. occidentalis são freqüentes. Apesar de próximo ao córrego Christone, no córrego Água da Veada não foram identificados espécimes de B. glabrata. A B. tenagophila é predominante nos córregos Bela Vista e São Luiz, localizados na micro-região de Ipauçu. A B. peregrina é uma espécie eventual, encontrada nesse estudo preferencialmente em um tanque de água abastecido por uma nascente.

Principalmente o córrego Christone/Ourinhos, localizado às margens do Rio Pardo, apresenta maior riqueza taxonômica de planorbídeos do gênero Biomphalaria (Figura 4a, b). Essa acentuada biodiversidade pode estar relacionada com a complexidade deste habitat constituído por latossolo, com abundante vegetação aquática flutuante, principalmente aguapés-Eichornia $S P$, um microambiente que serve de abrigo e suporte para desovas ovipositadas por caramujos (Freitas 1976) (Figura 5a). Considerando que a Biomphalaria glabrata é uma espécie nativa (França 2007), o padrão de distribuição da espécie pode estar correlacionada com sua preferência por habitats antropogênicos, ricos em matéria orgânica. (Bini et al. 1999, Abílio et al. 2006)

A baixa diversidade de espécies encontrada nos córregos de Ipauçu e a alta predominância da espécie B. tenagophila, (Figura 4a, b) podem estar correlacionadas com a natureza arenosa do substrato. Tipicamente instável, o substrato está sujeito a modificações na correnteza, possuindo baixa disponibilidade de matéria orgânica, que culmina na baixa oferta de alimento (Figura 5b). É interessante fazer referência aos dados geomorfológicos que mostram que Ipauçu está assentada num afloramento erodido, sob rochas do arenito original (Janasi et al. 2007a, b).É razoável supor que espécies de caramujos 

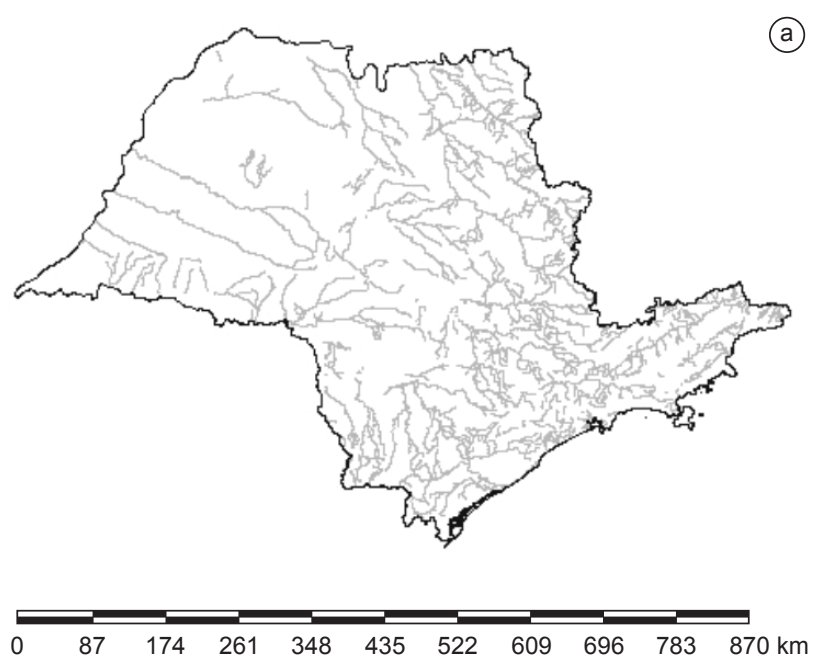

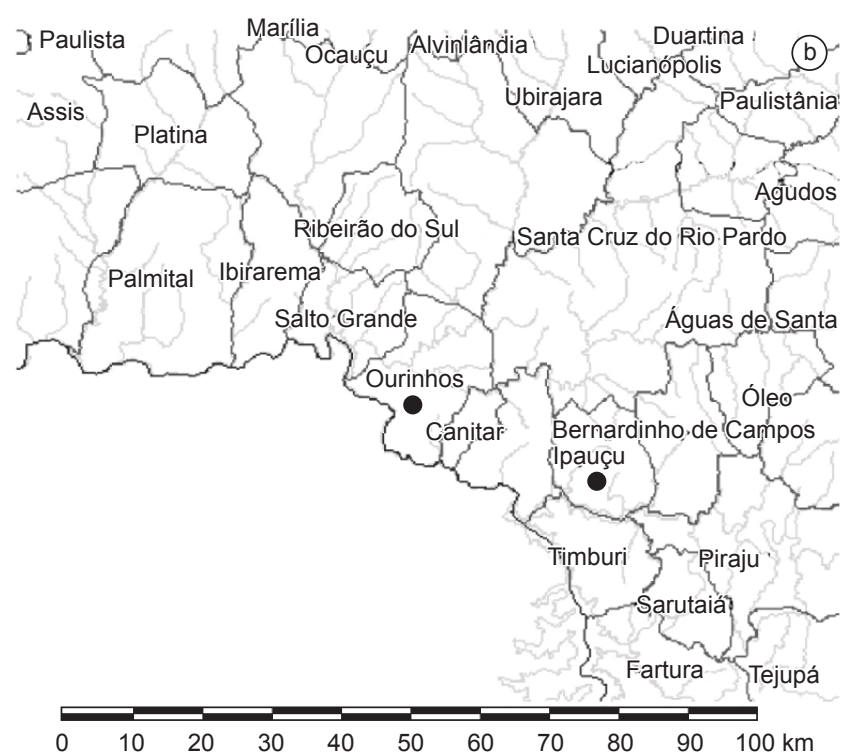

Figura 1. a) Localização panorâmica da área de estudo no estado de São Paulo, b) localização das micro-regiões Ourinhos e Ipauçu e c) dos córregos Christone, Água da Veada, Furnas e Jacu em Ourinhos.

Figure 1. a) Geographical location of the medium Paranapanema region at São Paulo state, Brazil, b) Ourinhos and Ipauçu microregions and c) Ourinhos streams.

que desenvolveram estratégias eficientes de sobrevivência ao stress ambiental colonizem preferencialmente um ecótopo deste tipo. Dentre as várias espécies de caramujos do gênero Biomphalaria, a B. tenagophila sobrevive longo tempo em dessecação, uma diapausa fisiológica que permite a recolonização do mesmo habitat até sessenta dias apos uma seca drástica ou uma baixa disponibilidade de alimento (Tuan \& Simões 1989).

Os resultados apresentados mostram que a distribuição de espécies de caramujos do gênero Biomphalaria é descontínua mesmo num espaço micro-geográfico. A análise genética feita preliminarmente 
Tuan, R

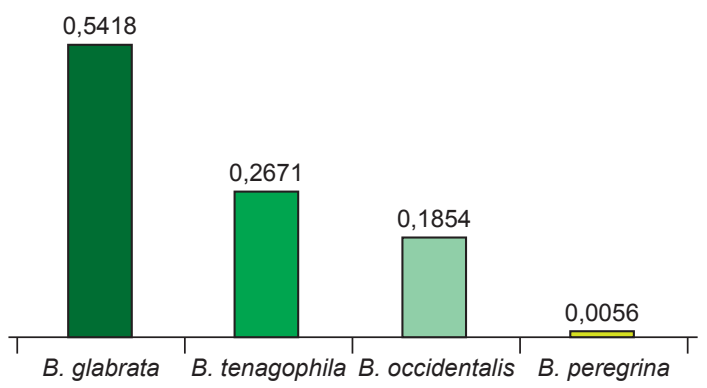

Figura 2. Frequência relativa de espécies de Biomphalaria nas micro-regiões Ourinhos e Ipauçu (São Paulo, SP, Brasil).

Figure 2. Relative frequency of Biomphalaria species in Ourinhos and Ipauçu micro-regions at S. Paulo state, Brazil.

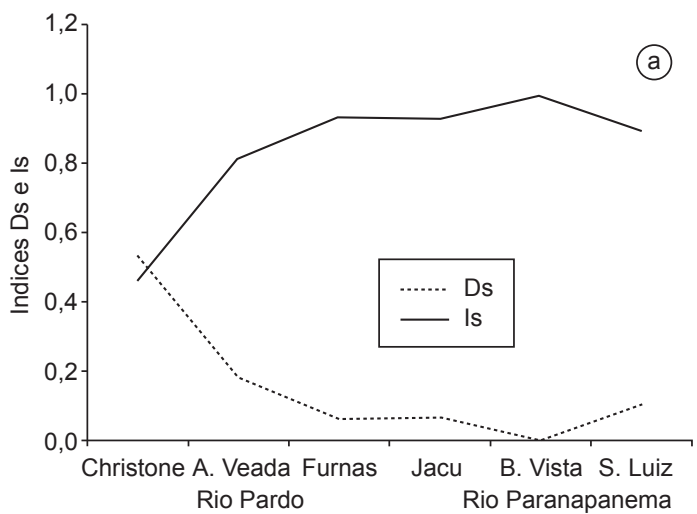

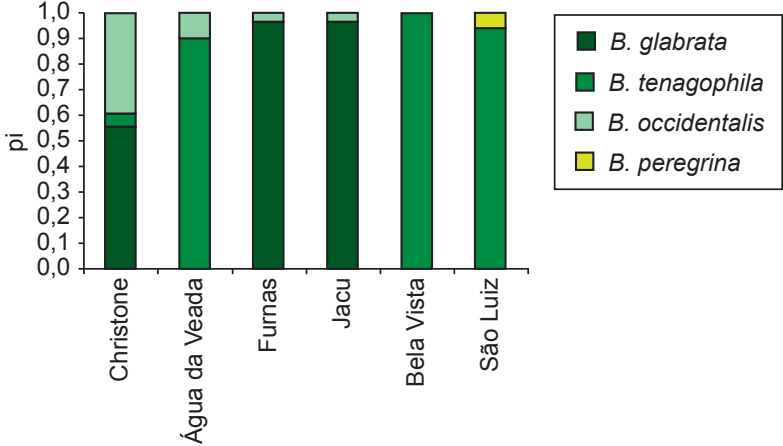

Figura 3. Frequência relativa de espécies de Biomphalaria nas coleções de água de Ourinhos e Ipauçu.

Figure 3. Relative frequency of Biomphalaria species in each stream at Ourinhos and Ipauçu microregions.

Figura 4. Índices de a) Diversidade e Dominância de Simpson e b) Equitabilidade Hill modificado (b).

Figure 4. Diversity, a) dominance and b) Hill equitability index (b) from Ourinhos and Ipauçu regions.
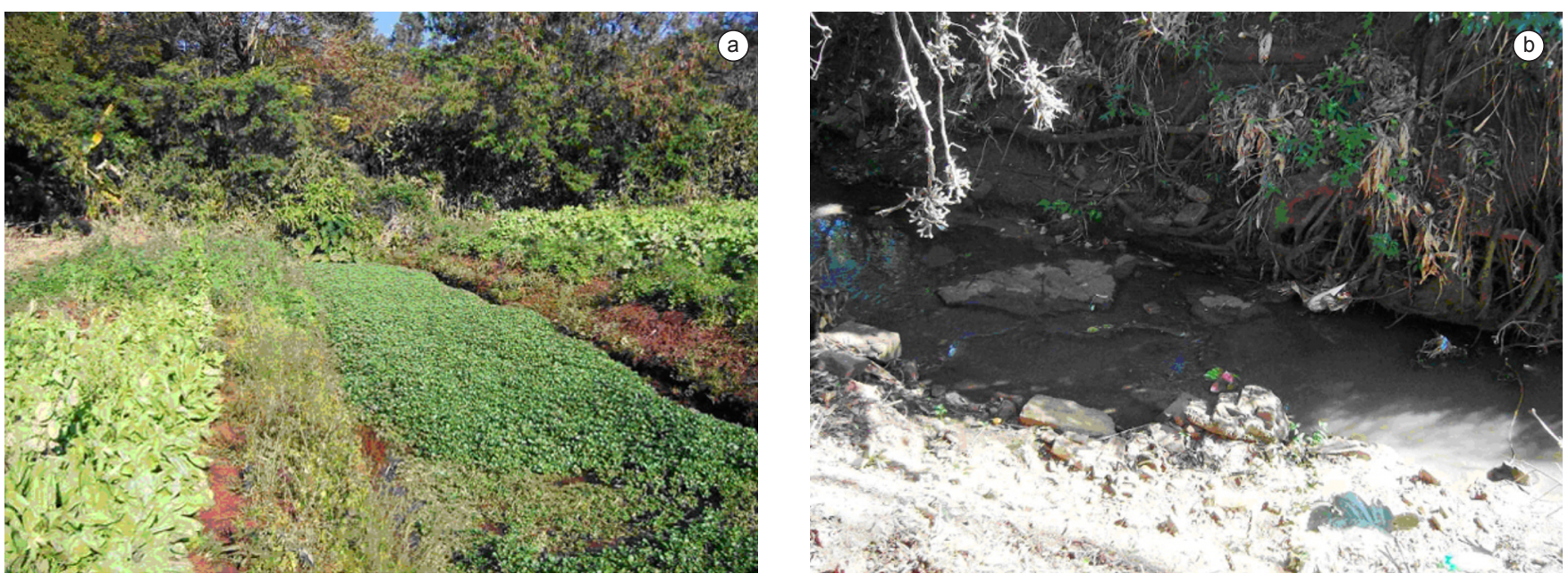

Figura 5. Caracterização geral dos córregos a) Christone em Ourinhos e b) São Luiz em Ipauçu.

Figure 5. General characterization of a) Christone and b) Ipauçu streams.

em populações de B. tenagophila Ipauçu e Ourinhos (Tuan \& Santos 2007), mostra diferenças significativas nos níveis de polimorfismos genético destas populações. Este resultado enfatiza a possibilidade de significativa fragmentação de uma população de caramujos de uma

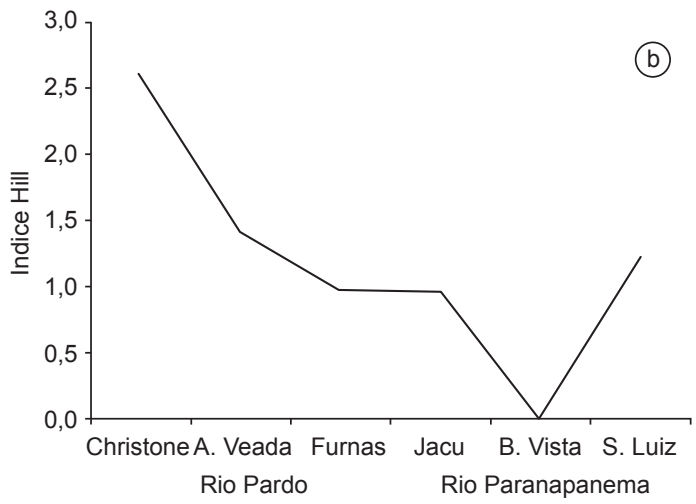

(b) mesma espécie. Se racionalmente considerarmos como heterogêneas as populações de caramujos de uma mesma espécie de Biomphalaria, alem de geneticamente distintas, seria importante analisar o impacto dessas diferenças em relação à suscetibilidade ao Schistosoma mansoni. 


\section{Referências Bibliográficas}

ABILIO, F.J.P., FONSECA-GESSNER, A.A., LEITE, R.L., RUFFO, T.L.M 2006. Gastrópodes e outros invertebrados do sedimento e associados à macrófita Eichhornia crassipes de um açude hipertrófico do semi-árido paraibano. BioTerra, 1(supl.):165-178

BINI, L.M., THOMAZ, S.M., MURPHY, K.J. \& CAMARGO, A.F.M. 1999. Aquatic macrophyte distribution in relation to water and sedimentconditions in the Itaipu Reservoir, Brazil. Hydrobiologia, 415(0): 147-154.

BRASIL. Ministério da Saúde. 2007. Vigilância e controle de moluscos de importância epidemiológica. Diretrizes técnicas: Programa de Vigilância e Controle da Esquistossomose (PCE). Brasília, DF

BUCKLING, A., KASSEN, R.; BELL, G. \& RAINEY, P.B. 2000. Disturbance diversity in experimental microcosms. Nature, 408(6815):961-964.

DESLANDES, N. 1959. Técnica de dissecção e exame de planorbídeos. Rev. Serv. Espec. Saúde Publica, 4(2):371-382.

ESTRADA, V.E., VELÁSQUEZ, L.E., CALDEIRA, R.L., BEJARANO, E.E., ROJAS, W. \& CARVALHO, O.M. 2006. Phylogenetics of South American Biomphalaria and description of a new species (Gastropoda: Planorbidae). J. Mollus. Stud. 72(3):221-228.

FRANÇA, R.S., SURIANI, A.L. \& ROCHA, O. 2007. Composição das espécies de moluscos bentônicos nos reservatórios do baixo rio Tietê (São Paulo, Brasil) com uma avaliação do impacto causado pelas espécies exóticas invasoras. Rev. Bras. Zool. 24(1): 41-51.

FREITAS, J.R. 1976. Ecologia de Vetores de Doenças, o Habitat Primitivo da Biomphalaria glabrata. Cienc. Cultura 28 (2): 212-217.

GERARD, C., CARPENTIER, A. \& PAILLISSON, J.M. 2008. Long-term dynamics and community structure of freshwater gastropods exposed to parasitism and other environmental stressors. Freshwater Biology, 53(3):470-484

JANASI, V.A., MONTANHEIRO, T.J., FREITAS, V.A., REIS, P.M., NEGRI, F.A. \& DANTAS, F.A. 2007b. Geology, petrography and geochemistry of the acid volcanism of the Paraná Magmatic Province in the Piraju-Ourinhos region, SE Brazil. Revista Brasileira de Geociências, 37(4):745-759.

JANASI, V.A., NEGRI, F.A., MONTANHEIRO, T.J., FREITAS, V.A., ROCHA, B.C. \& REIS, P.M. 2007a. Geochemistry of the eocretacic basalt magmatism in the Piraju-Ourinhos region, SE Brazil, and implications to the stratigraphy of the Serra Geral Formation. Revista Brasileira de Geociências, 37(1):148-162.

KAWAZOE, U., MAGALHÃES, L., HOTTA, L.K. \& TAKAKU, L. 1980. Competição biológica entre Biomphalaria glabrata (Say, 1818) e Biomphalaria tenagophila (Orbigny, 1835), em criadouros naturais no município de Ourinhos, SP, (Brasil). Rev. Saúde Publ. S.Paulo, 14(1):65-87.
MAGALHÃES, L.E. \& DIAS, L.C.Z. 1973. Estudo da suscetibilidade da Biomphalaria glabrata de Ourinhos (SP) à infecção pelo Schistosoma mansoni de Belo Horizonte (MG) e São José dos Campos (SP). Rev. Saúde Pública, S.Paulo, 7(3):295-297.

PARAENSE, W.L. 1955. Autofecundação e fecundação cruzada em Australorbis glabratus. Mem. Inst. Oswaldo Cruz, 53(2-4): 276-284.

PARAENSE, W.L. 1975. Estado atual da sistemática dos planorbídeos brasileiros. Arq. Mus. Nac. 55:105-111.

PARAENSE, W.L. 1981. Biomphalaria occidentalis sp.n. from South America (Mollusca: Basommatophora: Pulmonata). Mem Inst. Oswaldo Cruz, 76(2):199-211.

PIZA, J.T., RAMOS, A.S., MORAES, L.V.C., CORREA, R.R., TAKAKU, L. \& PINTO, A.C.M. 1972. Carta Planorbídica do Estado de São Paulo. Secretaria da Saúde do Estado de São Paulo, São Paulo. Campanha de Combate à Esquistossomose.

REY, L. 1952. Primeiro encontro de planorbídeos naturalmente inferstados por furcocercárias de $S$. mansoni no planalto paulista (Ourinhos). Rev Clin. S. Paulo, 28(5-6):57-64.

RODRIGUES, W.C. DivEs - Diversidade de espécies. Versão 2.0. Software e Guia do Usuário, 2005. Disponível em: <http://www.ebras.bio.br> Acesso em: 02 nov. 2005.

RUIZ, J.M. 1952. Índices cercáricos específicos do Schistosoma mansoni verificados em Neves e Mariana, Estado de Minas Gerais. Mem. Inst. Butantan, São Paulo, 24:63-68.

SHANON, C.E. 1948. A mathematical theory of communication. Bell Syst. Techn. J. 27(jul-oct):379-423,623-656.

SIMPSON, E.H. 1949. Measurement of diversity. Nature, 163(4148):688.

TELES, H.M.S. \& VAZ, J.F. 1987. Distribuição de Biomphalaria glabrata (Say, 1818) (Pulmonata, Planorbidae) no estado de São Paulo, Brasil. Rev. Saúde Pública, 21(6):508-512.

TELES, H.M.S. 2005. Distribuição geográfica das espécies dos caramujos transmissores de Schistosoma mansoni no Estado de São Paulo. Rev. Soc. Bras. Méd. Trop. 38(5):426-432.

TUAN, R. \& SANTOS P. 2007. ITS2 variability of Biomphalaria (Mollusca, Planorbidae) species from the Paranapanema Valley (São Paulo State, Brazil): diversity patterns, population structure, and phylogenetic relationships. Genet. Mol. Biol. 30(1):139-144.

TUAN, R. \& SIMÕES, L.C.G. 1998. Effect of self-fertilization on Biomphalaria tenagophila (Orbigny, 1835) (Pulmonata: Planorbidae). Genet. Mol. Biol. 21(4):477-478.

VAZ, J.F., TELES, H.M.S. \& TAKAKU, L. 1985. Levantamento planorbídico do estado de São Paulo: $7^{\text {a }}$ Região administrativa. Cienc. Cult. 37(12):2057-2062.

Recebido em 27/07/08

Versão reformulada recebida em 23/12/08

Publicado em 24/03/09 
